Alice Rosa da Silva

alicers@mecanica.ufu.br

Aristeu da Silveira Neto

aristeus@mecanica.ufu.br

Antonio Marcos G. de Lima

amglima@mecanica.ufu.br

Domingos Alves Rade

domingos@ufu.br

Federal University of Uberlândia

School of Mechanical Engineering

P.O.Box 593

38400-902 Uberlândia, MG, Brazil

\section{Numerical Simulations of Flows over a Rotating Circular Cylinder Using the Immersed Boundary Method}

In this paper, numerical simulations of incompressible flows around rotating circular cylinders have been performed. The two-dimensional Navier-Stokes equations are solved by using a Cartesian non-uniform grid. The Immersed Boundary Method (IBM) with the Virtual Physical Model (VPM) was used in order to model the presence of the circular cylinder in the flow. The fractional time step method was used to coupling the pressure and velocity fields. The simulations were carried out for Reynolds numbers equals to 60, 100 and 200 for different specific rotations. The effects of rotation on flow characteristics and fluctuating forces were investigated. The Strouhal number, obtained by performing the Fast Fourier Transform (FFT) of the temporal distribution of the lift coefficient, and the pressure coefficients, were also been calculated. Vorticity contours are presented considering different values of the Reynolds number and specific rotation. The numerical results obtained are compared to those obtained by other authors and the usefulness of the numerical methodology composed by the combination of the IBM with the VPM to simulate flows in the presence of mobile bodies is highlighted.

Keywords: immersed boundary method, virtual physical model, rotating cylinder, vortex shedding

\section{Introduction}

Incompressible viscous flow around circular cylinders is a classical problem in fluid mechanics that has been a topic of interest both numerically and experimentally by many authors (Lai and Peskin, 2000; Ryan et al., 2004; Su et al., 2006). Flows over a bundle of cylinders have been also extensively studied and it can be cited cylinders side by side, in tandem and in staggered configurations (Deng et al., 2006; Sumner et al., 2007; Alam and Zhou, 2007; Lima e Silva et al., 2007; da Silva et al., 2009). Also, a number of studies have been proposed in order to investigate the effects of rotating circular cylinders on the wake structure (Juncu, 2007).

The dynamic of flows passing over rotating circular cylinders is different from those observed for stationary cylinders. The rotation of a cylinder in a flow is expected to modify the wake of the flow and the vortex shedding configuration which may affect the lift and drag forces. The effective control of drag force exerted by the fluid on the cylinder has been a challenge which has received a particular attention in the last decades. The drag force on the cylinder may be reduced by an active control in the rotation of the cylinder. Aldoss and Abou-Arab (1990) have investigated experimentally the flow around a rotating cylinder placed in a cross-flow air stream. They obtained the velocity profiles, the skin friction coefficient, the stagnation and the separation points for different values of the specific rotation, which varied from 0 to 1.25 . Kang et al. (1999) presented a numerical study of two-dimensional laminar flow over a rotating circular cylinder by taking into account a constant angular velocity, with the aim of controlling the vortex shedding and better understanding the flow dynamic. The numerical simulations were performed for flows by taking into account three different values of Reynolds numbers: 60,100 and 160 in the range of $0 \leq \alpha \leq 2.5$, where $\alpha$ is the dimensionless specific rotation. In their work, the momentum and continuity equations were transformed by introducing generalized coordinates and volume fluxes across the faces of the cell. These transformed equations were integrated in time by using a fully-implicit fractional step method, composed of four-step time splitting. Also, the cross-derivative diffusion terms were advanced over the time by using the Crank-Nicolson method and over the space by applying the second-order central difference

Paper accepted October, 2010. Technical Editor: Francisco Ricardo Cunha scheme. Finally, a Newton method has been used in order to solve the discretized nonlinear equations.

Nair et al. (1998) investigated flows around a circular cylinder in translation and rotation at different Reynolds numbers and different specific rotations. According to the authors, the results obtained at Reynolds number 200 and at low specific rotation, 0.5 and 1.0, have been compared well with experimental visualizations. Dol et al. (2007) have studied experimentally the turbulent wake of a rotating circular cylinder at Reynolds numbers equal to 9000 by considering a specific rotation between 0 and 2.7. They verified, through hot wire anemometry analysis, that the periodic vortex shedding near the body is suppressed for specific rotations greater than 2.0. They have also observed that the Strouhal number and the advective speed of the vortices increase with the specific rotation until the suppression of the wake. There are many other applications areas of this kind of study, as the control of the shear layer in airfoils (Badr et al., 1989), in projects of aerodynamics of vehicles and design of heat exchangers. A recent particular emphasis appears from the need to study the three-dimensional effects of the wake behind bodies. Such effects are important when the force integrated over the body is considered, especially in the context of Vortex Induced Vibration (VIV) (Williamson, 1997).

This paper is dedicated to the use of the Immersed Boundary Methodology combined with the Virtual Physical Model to simulate flows over a moving circular cylinder in order to analyze the effects of the rotation on the flow and to better understand the mechanism of vortex shedding suppression. This is accomplished by the presentation of the instantaneous flow fields, the lift and the drag coefficients, the Strouhal number and the pressure distributions. The numerical simulations were carried out for flows at Reynolds numbers 60, 100 and 200 and for specific rotation in the range of $0 \leq \alpha \leq 3.0$.

$$
\begin{aligned}
& \text { Nomenclature } \\
& \qquad \begin{array}{cl}
C_{d} & =\text { drag coefficient }\left[F_{d} /\left(0.5 \rho U^{2} d\right)\right], \text { dimensionless } \\
C_{l} & =\text { lift coefficient }\left[F_{l} /\left(0.5 \rho U^{2} d\right)\right] \text {, dimensionless } \\
d & =\text { diameter of the cylinder, } m \\
D & =\text { distribution function, } m^{-2}
\end{array}
\end{aligned}
$$




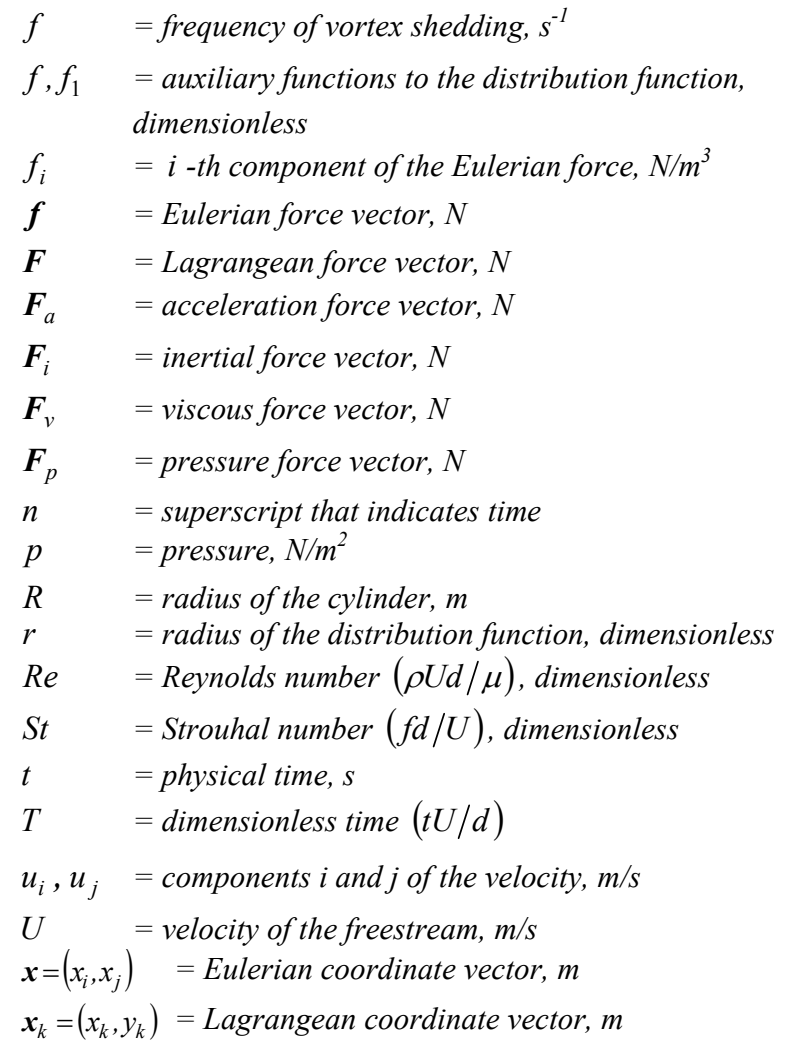

\section{Greek Symbols}

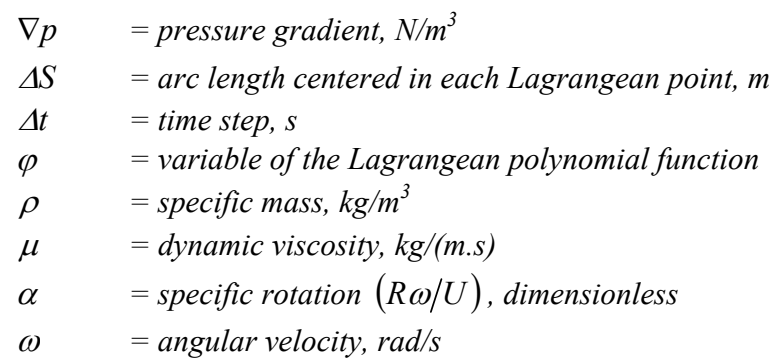

\section{Mathematical Methodology}

The simulations that follow have been performed using the Immersed Boundary Method (Peskin, 1977) combined with the Virtual Physical Model (Lima e Silva et al., 2007). This methodology is based on the Navier-Stokes equations with an added force source term which acts upon the fluid so that the particle perceives the existence of the solid interface and makes the exchange of information between the fluid and the solid. In this methodology a mixed Eulerian-Lagrangean formulation is used, where an Eulerian grid (fixed) describes the flow and a Lagrangean grid (it can be fixed or not) describes the immersed bodies. These meshes are geometrically independent allowing the study of the flows around simple, complex, movable and deformable geometries without the remeshing process. These two formulations are coupled by a force field obtained at the Lagrangean points and then distributed over the Eulerian nodes in the body neighborhood. Figure 1 depicts these grids for a two-dimensional domain with an arbitrary interface.

\section{Mathematical Formulation for the Fluid}

The two-dimensional viscous and incompressible flows can be modeled by using the following Navier-Stokes equations:

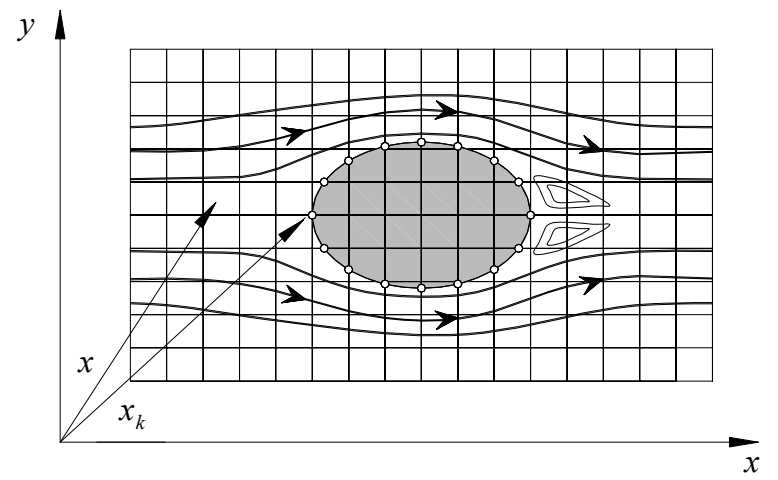

Figure 1. Illustration of the Eulerian and Lagrangean grids.

$$
\begin{aligned}
& \frac{\partial u_{i}}{\partial t}+\frac{\partial\left(u_{i} u_{j}\right)}{\partial x_{j}}=-\frac{1}{\rho} \frac{\partial p}{\partial x_{i}}+\frac{\partial}{\partial x_{j}}\left[v\left(\frac{\partial u_{i}}{\partial x_{j}}+\frac{\partial u_{j}}{\partial x_{i}}\right)\right]+f_{i} \\
& \frac{\partial u_{i}}{\partial x_{i}}=0
\end{aligned}
$$

where $\rho\left[\mathrm{kg} / \mathrm{m}^{3}\right]$ and $v\left[\mathrm{~m}^{2 / \mathrm{s}}\right]$ are the specific mass and the kinematic viscosity, respectively; $u_{i}[\mathrm{~m} / \mathrm{s}]$ is $i$-th component of the velocity, $p\left[N / m^{2}\right]$ is the pressure and $f_{i}\left[N / m^{3}\right]$ is the $i$-th component of the Eulerian force, which is calculated by the distribution of the components of the Lagrangean force vector $\boldsymbol{F}\left(\boldsymbol{x}_{k}\right)[N]$ according to Eq. (3). The Eulerian force term exists in the Eulerian points coincident or close to the Lagrangean grid, being zero for the other points of the domain.

$$
\boldsymbol{f}(\boldsymbol{x})=\sum_{k} D_{i j}\left(\boldsymbol{x}-\boldsymbol{x}_{k}\right) \boldsymbol{F}\left(\boldsymbol{x}_{k}\right) \Delta S^{2}\left(\boldsymbol{x}_{k}\right)
$$

In Eq. (3) $\boldsymbol{x}[\mathrm{m}]$ and $\boldsymbol{x}_{k}[\mathrm{~m}]$ are the position vectors of the Eulerian and Lagrangean points, respectively, and $\Delta S[\mathrm{~m}]$ is the arc length centered in each Lagrangean point as illustrated in Fig. 2(a). It is worth to mention that the Lagrangean points are evenly spaced. $D_{i j}\left[\mathrm{~m}^{-2}\right]$ is the interpolation/distribution function proposed by Peskin and McQueen (1994):

$$
D_{i j}\left(\boldsymbol{x}_{k}\right)=\frac{f\left[\left(x_{k}-x_{i}\right) / h\right] f\left[\left(y_{k}-y_{i}\right) / h\right]}{h^{2}}
$$

(4)

$$
f(r)=\left\{\begin{array}{l}
f_{1}(r) \quad \text { if } \quad\|r\| \leq 1 \\
\frac{1}{2}-f_{1}(2-\|r\|) \text { if } \quad 1<\|r\|<2 \\
0 \text { if }\|r\|>2
\end{array}\right.
$$

where $f_{1}(r)=\left(3-2\|r\|+\sqrt{1+4\|r\|-4\|r\|^{2}}\right) / 8$ and $r$ are the influence radius of the distribution function which can be computed as $\left(x_{k}-x_{i}\right) / h$ or $\left(y_{k}-y_{i}\right) / h$, depending on the direction in which the property is distributed. $h$ is the size of the Eulerian grid and $\left(x_{i}, y_{i}\right)$ represents the Eulerian coordinates of the point. 
The distribution force represented by Eq. (3) is computed over the grids that are located at the maximum distance of two grids. Despite the given idea of discontinuous force field, the distribution allows obtaining a smooth field without steps. This is due to the fact that as away from the interface, the distribution function gives lower values, tending to zero.
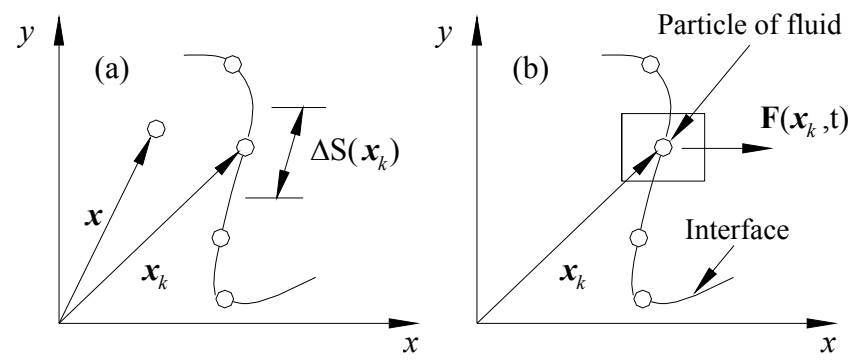

Figure 2. (a) Illustration of the arc length centered in each Lagrangean point and the vectors $x$ and $x_{k} ;(b)$ particle of fluid on the interface.

\section{Mathematical Formulation for the Fluid-Solid Interface - Virtual Physical Model}

The Virtual Physical Model enables to calculate the Lagrangean force field based on the momentum balance over a particle of fluid placed on the Lagrangean points, as shown in Fig. 2(b). By applying the Newton's second law to such a particle of fluid, the Lagrangean force is found as follows:

$$
\begin{aligned}
\boldsymbol{F}\left(\boldsymbol{x}_{k}, t\right) & =\underbrace{\rho \frac{\partial \boldsymbol{V}\left(\boldsymbol{x}_{k}, t\right)}{\partial t}}_{\boldsymbol{F}_{a}}+\underbrace{\rho \nabla\left[\boldsymbol{V}\left(\boldsymbol{x}_{k}, t\right) \boldsymbol{V}\left(\boldsymbol{x}_{k}, t\right)\right]}_{\boldsymbol{F}_{i}}+\underbrace{\nabla \boldsymbol{p}\left(\boldsymbol{x}_{k}, t\right)}_{\boldsymbol{F}_{p}}- \\
& -\underbrace{\mu \nabla\left[\nabla \boldsymbol{V}\left(\boldsymbol{x}_{k}, t\right)+\nabla^{T} \boldsymbol{V}\left(\boldsymbol{x}_{k}, t\right)\right]}_{\boldsymbol{F}_{v}}
\end{aligned}
$$

In Eq. (6), the terms $\boldsymbol{F}_{a}[N], \quad \boldsymbol{F}_{i}[N], \boldsymbol{F}_{v}[N]$ and $\boldsymbol{F}_{p}[N]$ are evaluated over the interface points using the pressure and velocity fields, calculated in Eulerian mesh through interpolation. In addition, these calculations take into account that at the interface, the fluid velocity must be equal to the interface velocity due to no-slip boundary condition. The spatial derivatives of pressure and velocity are calculated from the velocity and pressure obtained by the momentum and continuity equations. The derivatives that compose these terms are computed by performing a second-order Lagrange polynomials. Equations (7) and (8) are given for a generalized variable $\varphi$, which can represent the velocity components and the pressure. Once obtained the force given by Eq. (6), it is distributed to Eulerian nodes according to Eq. (3) in order to obtain the Eulerian force field that accounts for the immersed bodies.

$$
\begin{aligned}
\frac{\partial \varphi}{\partial x}\left(x_{k}, y_{k}\right)= & \frac{\left(x_{k}-x_{2}\right)}{\left(x_{1}-x_{2}\right)\left(x_{1}-x_{k}\right)} \varphi_{1}+\frac{\left(x_{k}-x_{1}\right)}{\left(x_{2}-x_{1}\right)\left(x_{2}-x_{k}\right)} \varphi_{2}+ \\
& +\frac{\left(x_{k}-x_{1}\right)+\left(x_{k}-x_{2}\right)}{\left(x_{k}-x_{1}\right)\left(x_{k}-x_{2}\right)} \varphi_{k} \\
\frac{\partial^{2} \varphi}{\partial x^{2}}\left(x_{k}, y_{k}\right) & =\frac{2 \varphi_{1}}{\left(x_{1}-x_{2}\right)\left(x_{1}-x_{k}\right)}+\frac{2 \varphi_{2}}{\left(x_{2}-x_{1}\right)\left(x_{2}-x_{k}\right)}+ \\
& +\frac{2 \varphi_{k}}{\left(x_{k}-x_{1}\right)\left(x_{k}-x_{2}\right)}
\end{aligned}
$$

It is important to note that the derivatives in the $y$ direction can be obtained in the similar way. The velocity components and pressure are interpolated by using auxiliary points $x_{i}$, with $i=1,2$, 3 and 4 disposed in $x$ and $y$ directions. After, equations (7) and (8) are used to evaluate the terms appearing in Eq. (6).

\section{Numerical Methodology}

The momentum and continuity equations are solved by using the finite difference method through the fractional step method (Chorin, 1968) which is based on the pressure correction concept. Using the initials field of velocity, pressure and force, an estimated velocity field is obtained. These estimated fields are used to calculate the pressure correction by solving a linear system for which the Modified Strongly Implicit Procedure (MSI), developed by Scheneider and Zedan (1981), is applied. The Poisson equation gives the coupling between the Eqs. (1) and (2). That equation also provides the values of pressure that allow the velocity components obtained by solving the Navier-Stokes equations satisfy the mass conservation. The time discretization was done by the second order Runge-Kutta method.

An estimation of the velocity is calculated as follows:

$$
\begin{aligned}
\frac{\tilde{u}_{i}^{n+1}-u_{i}^{n}}{\Delta t} & =-\frac{1}{\rho} \frac{\partial p^{n}}{\partial x_{i}}-\left[\frac{\partial\left(u_{i} u_{j}\right)}{\partial x_{j}}\right]^{n}+\frac{\partial}{\partial x_{j}}\left[v\left(\frac{\partial u_{i}}{\partial x_{j}}+\frac{\partial u_{j}}{\partial x_{i}}\right)\right]^{n}+ \\
& +f_{i}^{n}
\end{aligned}
$$

where $\tilde{u}_{i}[\mathrm{~m} / \mathrm{s}]$ is the estimated velocity component, $\Delta t[\mathrm{~s}]$ is the computational time step and $n$ is the substep index. The Poisson equation for pressure correction, $p^{\prime n+1}$, with the source term given by the divergent of the estimated velocity, is given by:

$$
\nabla^{2} p^{n+1}=\rho \frac{\vec{\nabla} \overrightarrow{\tilde{u}}^{n+1}}{\Delta t}
$$

The velocity field is updated by solving the following equation:

$$
u_{i}^{n+1}=\tilde{u}_{i}^{n+1}-\frac{\Delta t}{\rho} \frac{\partial p^{\prime n+1}}{\partial x_{i}}
$$

The previous pressure field, $p^{n}$, and the correction pressure, $p^{\prime n+1}$, are used to calculate the updated values of the pressure field according to the following expression:

$$
p^{n+1}=p^{n}+p^{n+1}
$$

\section{Description of the Problem}

Figure 3(a) depicts the rectangular computational domain used in the numerical simulations that follows, and Fig. 3(b) illustrates the components of the velocity imposed over each Lagrangean point $k$, obtained by decomposing the tangential velocity $V_{t g}=\omega R$. The domain was set to be $L u+L d=40 d$ in the streamwise direction and $H=15 d$ in the cross-stream direction, where $L u=16.5 d$, $L d=23.5 d$ and $d$ is the diameter of the cylinder located inside 
the computational domain. The upper and lower boundaries are placed at $7.5 d$ from the center of the cylinder, and the flow direction is from the left to the right side of the domain. The boundary conditions adopted for the velocity are: inlet: $u=U$ and $v=0$; outlet: $\partial u / \partial x=\partial v / \partial x=0$; top and bottom: $\partial u / \partial y=\partial v / \partial y=0$.

For the pressure, the Newmann conditions in the inlet $(\partial p / \partial x=0)$, and the Dirichlet conditions in the outlet, top and bottom $(p=0)$, were assumed.
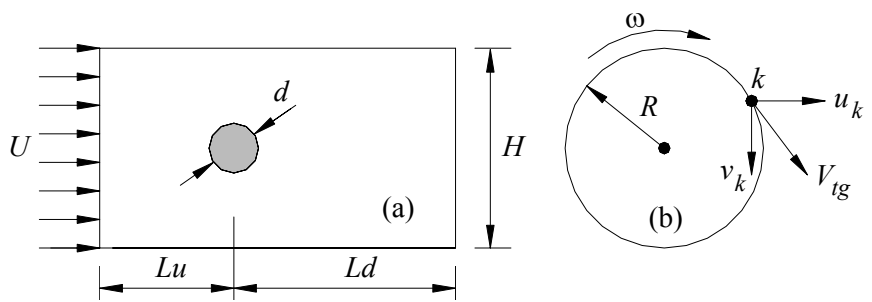

Figure 3. (a) Illustration of the computational domain with a single rotating cylinder; (b) velocities components and angular velocity.

The following damping function is used in the outlet of the domain (Meitz and Fasel, 2000) which acts over the fluctuations of the velocity in order to avoid that reflections propagate back into the fluid domain:

$$
g(\varepsilon)=1-6 \varepsilon^{5}+15 \varepsilon^{4}-10 \varepsilon^{3}
$$

with $\varepsilon=\left(i-i_{1}\right) /\left(i_{2}-i_{1}\right)$, where $i$ is the grid number; $i_{1}$ and $i_{2}$ $\left(i_{1} \leq i \leq i_{2}\right)$ are the initial and final grid points of the damped zone.

\section{Numerical Results}

The simulations were performed at Reynolds numbers 60,100 and 200. The time step was $\Delta t=0.00001 s$ at the first iteration, and it is increased gradually up to $\Delta t=0.001 \mathrm{~s}$ during the first 100 iterations. As the geometry of the interface is established by the force field, the time step is calculated in such a way that the $C F L \leq 1.0$ (Courant-Friedrichs-Lewy criterion) is attained. This condition is required to the stability of the explicit numerical scheme.

The grid used in the numerical simulations is composed of $200 \times 125$ points in $x$ and $y$ directions, respectively. The rotation of the cylinder was started after one physical second. Normally, at this time, the geometry of the cylinder is well defined, according to the characteristics of the IBM method. At the beginning of the simulations, $t=0 \mathrm{~s}$, the geometry is not well defined, which can be observed by the streamlines across the cylinder. After the prescribed time, the streamlines perfectly contour the cylinder, which means that it is being recognized by the fluid. The cylinder is accelerated according to prescribed angular velocity, which is used to calculate the tangential velocity as shown in Fig. 3(b).

It is important to mention that the time step and the refinement of the grid are aspects that influence the computational time required to generate the solutions. If the numerical simulations presented herein are performed in an Intel Core 2 Quad having $2.40 \mathrm{GHz}$ and $3.23 \mathrm{~GB}$ of RAM, the CPU time is approximately 2.5 hours. Moreover, other computational aspects such as the capability of parallelization process should be an interesting strategy that enables to reduce the computational burden, even for the case of more refined grids.

\section{Characteristics of Vortex Shedding}

Figures 4 to 7 show the instantaneous vorticity surfaces for different specific rotations $\alpha$ in order to verify the structure of the flow in the initial time at Reynolds number 200 . From Fig. 4 it can be seen that for the stationary cylinder, the clock-wise vorticity is negative and the counter-clock-wise is positive.

The time evolution of the flow at $\alpha=0.5$ is shown in Fig. 5. It is similar to that observed for stationary cylinder. However, the vortices take different form and size as the time evolves. The vortex formed in the inferior side of the cylinder grows faster than the vortex formed at the superior side of the cylinder, and it is advected after $7 \mathrm{~s}$. On the other hand, the negative vortex is advected at $t=9 \mathrm{~s}$. The wake is bended towards the clock-wise side following the rotation of the cylinder.

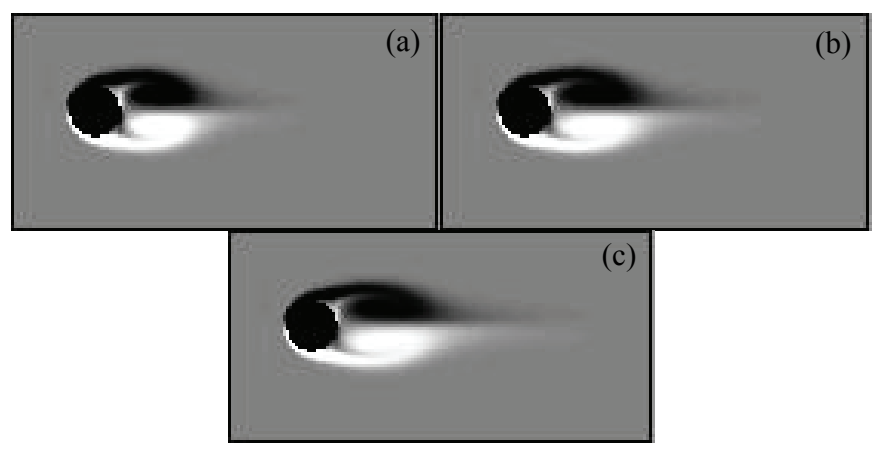

Figure 4. Time evolution of the vorticity contours for $\alpha=0$ and $\operatorname{Re}=200$ : (a) $\mathrm{t}=5 \mathrm{~s}$; (b) $\mathrm{t}=6 \mathrm{~s}$; (c) $\mathrm{t}=7 \mathrm{~s}$.

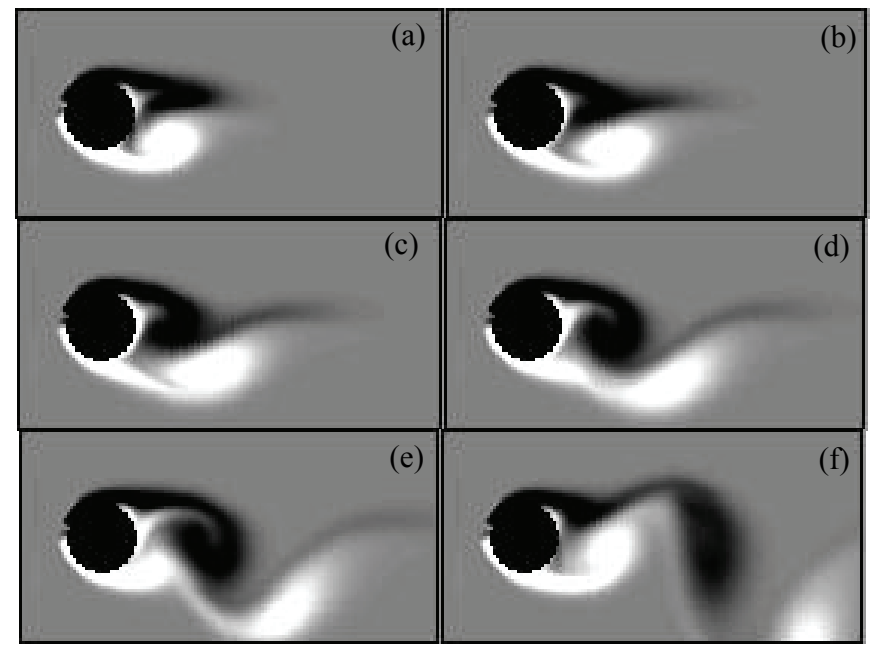

Figure 5. Time evolution of the vorticity contours for $\alpha=0.5$ and $\operatorname{Re}=200$ : (a) $\mathrm{t}=3 \mathrm{~s}$; (b) $\mathrm{t}=4 \mathrm{~s}$; (c) $\mathrm{t}=5 \mathrm{~s}$; (d) $\mathrm{t}=6 \mathrm{~s}$; (e) $\mathrm{t}=7 \mathrm{~s}$; (f) $\mathrm{t}=9 \mathrm{~s}$.

Figure 6 shows that at $\alpha=1.0$ for the time scaling from $t=3 \mathrm{~s}$ to $t=5 \mathrm{~s}$, the positive vortices have rounded form, while the negative vortex are elongated. The first vortex is advected after $t=4$ s. By increasing the specific rotation to $\alpha=1.5$ (see Fig. 7), it is not observed in the wake a relevant difference between the results obtained for $\alpha=1.0$. As the time progresses, the inclination of the wake becomes more pronounced for great values of the specific rotation. 


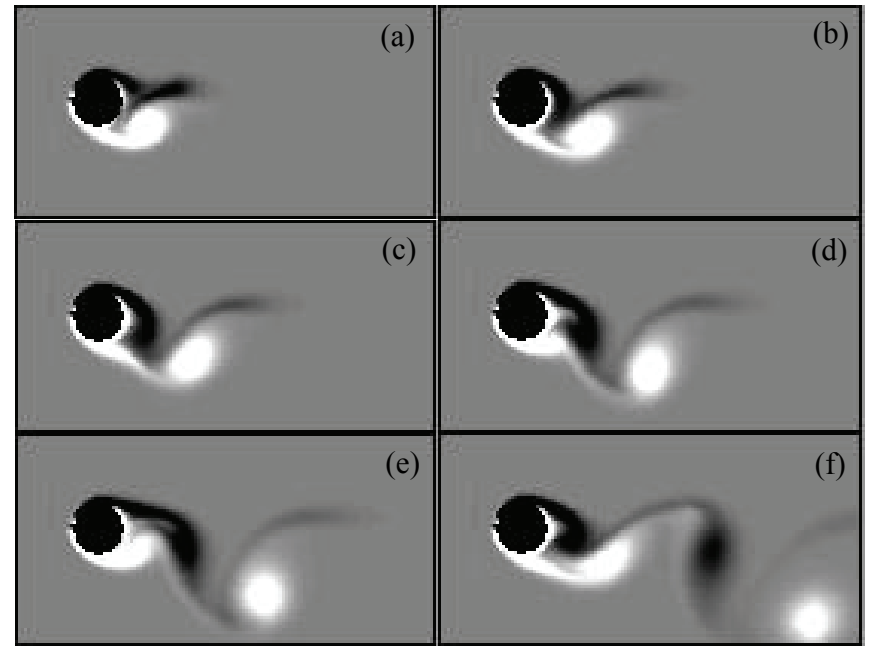

Figure 6. Time evolution of the vorticity contours for $\alpha=1.0$ and $\operatorname{Re}=200$ : (a) $t=3 \mathrm{~s}$; (b) $t=4 \mathrm{~s}$; (c) $t=5 \mathrm{~s}$; (d) $t=6 \mathrm{~s}$; (e) $t=7 \mathrm{~s}$; (f) $t=10 \mathrm{~s}$.

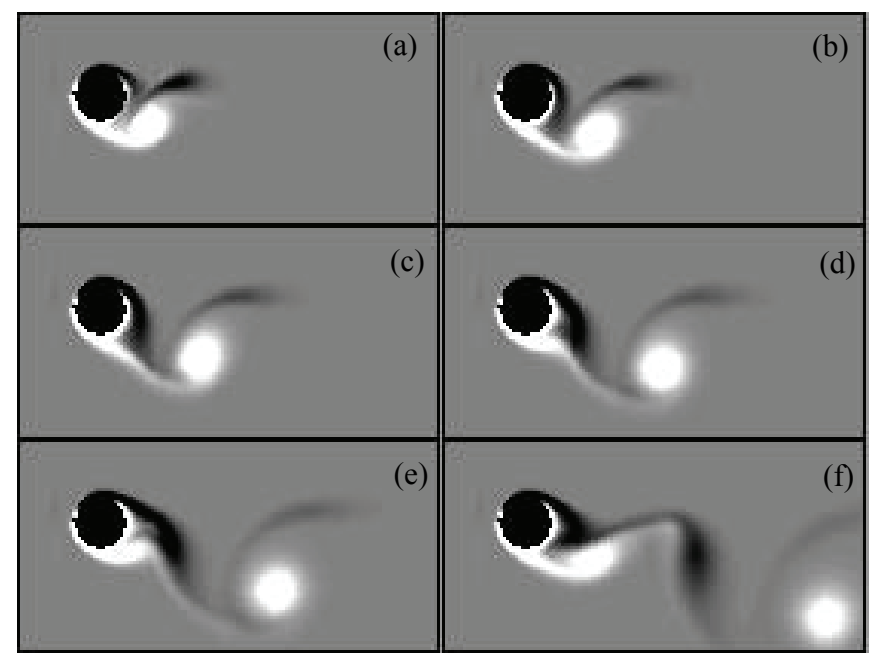

Figure 7. Time evolution of the vorticity contours for $\alpha=1.5$ and $\operatorname{Re}=200$ : (a) $t=3 \mathrm{~s}$; (b) $t=4 \mathrm{~s}$; (c) $t=5 \mathrm{~s}$; (d) $t=6 \mathrm{~s}$; (e) $t=7 \mathrm{~s}$; (f) $t=10 \mathrm{~s}$.

Figures 8 to 10 show the instantaneous vorticity fields for different Reynolds numbers, and for several values of the specific rotations. It can be seen the presence of vortices for small values of $\alpha$. The vortices tend to disappear as $\alpha$ increases and tend to the critical value $\alpha_{c}$, which depends on the Reynolds number. The process of vortices suppression can also be analyzed by the drag and lift coefficients, as discussed later. Also, it can be noted that for $\alpha=0$, the vortices wake is aligned with the horizontal direction for all Reynolds numbers, as expected. As the rotation increases the wake becomes more bended toward the cylinder rotation direction.

For all Reynolds number the vortex shedding process is eliminated by the rotating effects. The value of rotation for which the vortex street is suppressed is defined herein as critical rotation, $\alpha_{c}$. The $\alpha_{c}$ corresponding to the Reynolds numbers equals to 60,100 and 200 are, respectively, $1.4,1.8$ and 2.0. It is important to mention that the two first values of the critical rotation are in agreement with the results obtained by Kang et al. (1999) that have performed simulations for Reynolds numbers equal to 60,100 and 160 .

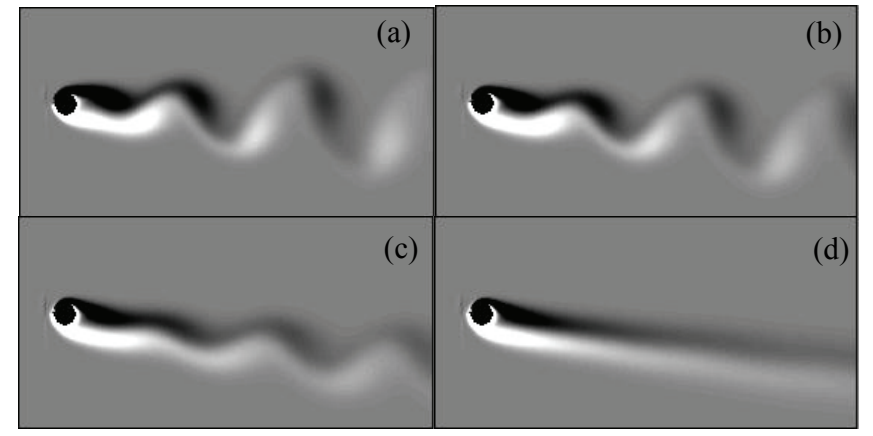

Figure 8. Vorticity contours at $t=200 \mathrm{~s}$ for $\operatorname{Re}=60$ : (a) $\alpha=0.5$; (b) $\alpha=1.0$; (c) $\alpha=1.4$; (d) $\alpha=1.5$.

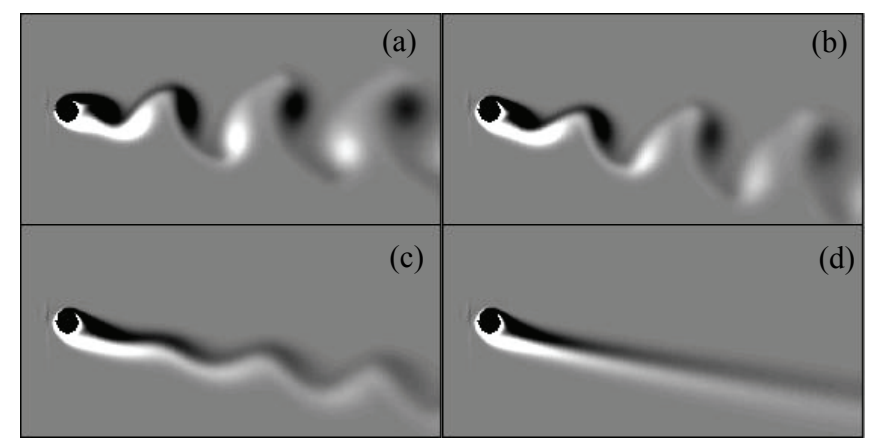

Figure 9. Vorticity contours at $t=200 \mathrm{~s}$ for $\operatorname{Re}=100$ : (a) $\alpha=0.5$; (b) $\alpha=1.5$; (c) $\alpha=1.85$; (d) $\alpha=2.0$.

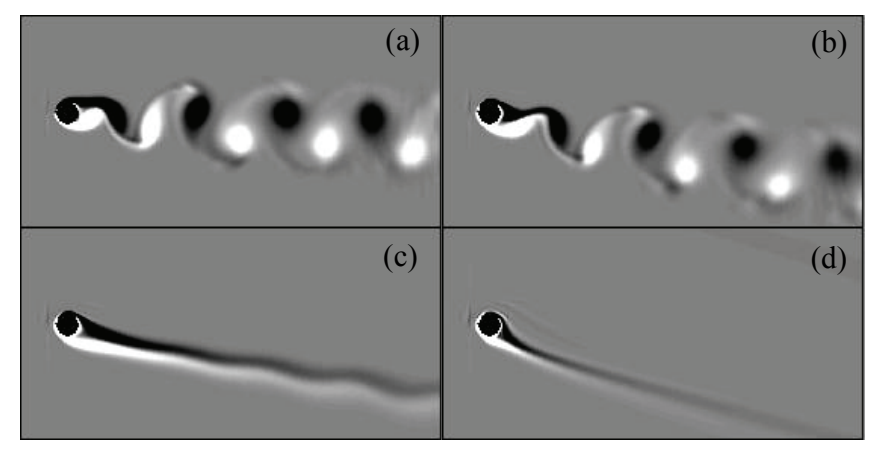

Figure 10. Vorticity contours at $t=200 \mathrm{~s}$ for $\operatorname{Re}=200$ : (a) $\alpha=0.5$; (b) $\alpha=1.5$; (c) $\alpha=2.0$; (d) $\alpha=3.0$.

\section{Qualitative Results by Streamlines}

Figure 11 shows the streamlines for flow past a rotating circular cylinder obtained in the present study for four different instants of time. The present results are compared with the numerical and experimental results obtained by Nair et al. (1998) and Countanceau and Menard (1985), respectively, for $R e=200$ and $\alpha=0.5$. The time scale adopted in the present computations is the same of that used by the cited authors. One can verify a good agreement of the flow topology when compared with the other works. Moreover, it can be observed the coherence of the structure in the presented results. Qualitatively, it can be concluded that the vortices obtained in the present work are in a similar position and have approximately the same size as those presented by the other authors. 


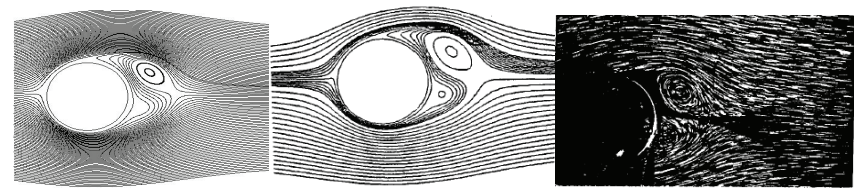

(a)

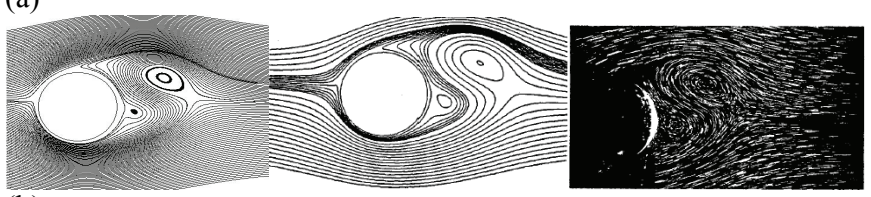

(b)

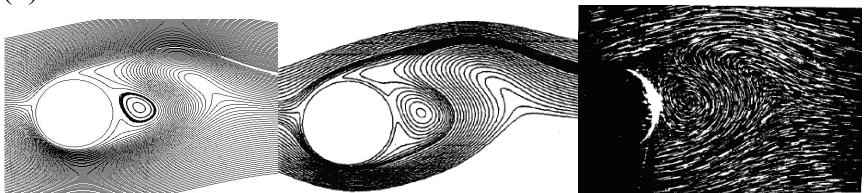

(c)

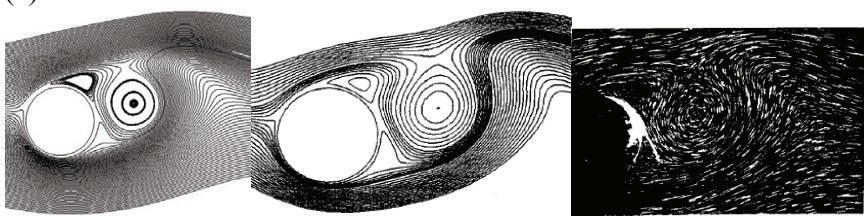

(d)

Figure 11. Present study (left column), numerical results by Nair et al. (1998) (middle column), and experimental results by Countanceau and Menard (1985) (right column) for (a) $t=1.5 \mathrm{~s}$, (b) $t=2.5 \mathrm{~s}$, (c) $t=3.5 \mathrm{~s}$ and (d) $\mathrm{t}=4.5 \mathrm{~s}$

\section{Pressure Distribution}

Figure 12 shows an illustrative scheme of the angle $\theta$ along the surface of the immersed body, which is defined as zero at the upstream point of the cylinder. The velocity of the particle increases from points $A$ to $B$, and decreases from points $B$ to $C$. On the other hand, the pressure decreases from $A$ to $B$ and increases from $B$ to $C$.

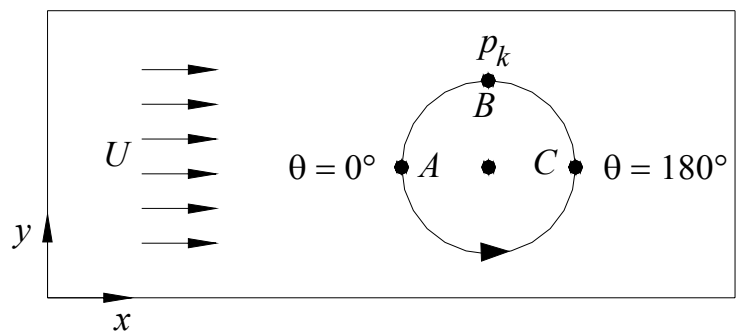

Figure 12. Illustration of the angle $\theta$ along the surface of the cylinder.

The pressure coefficient is obtained using the static pressure, $p_{k}$, at each Lagrangean point, which is calculated according to the following expression:

$$
C_{p}=\frac{p_{k}-p_{\infty}}{0.5 \rho U^{2}}
$$

where $p_{\infty}$ is the static pressure of the undisturbed flow, i.e., at the inlet of the domain, and the term $0.5 \rho U^{2}$ represents the dynamic pressure, which is taken as the reference pressure.

Figure 13 shows the mean pressure coefficients over the cylinder for $R e=200$ and for different specific rotations. This figure shows that the pressure distribution coefficient for $\alpha=0$ is almost symmetric for $0^{\circ} \leq \theta \leq 360^{\circ}$, as expected. However, for the rotating cases, it is shown that the pressure distribution is not symmetric, which corresponds to the non symmetric wake shown in the previous figures.

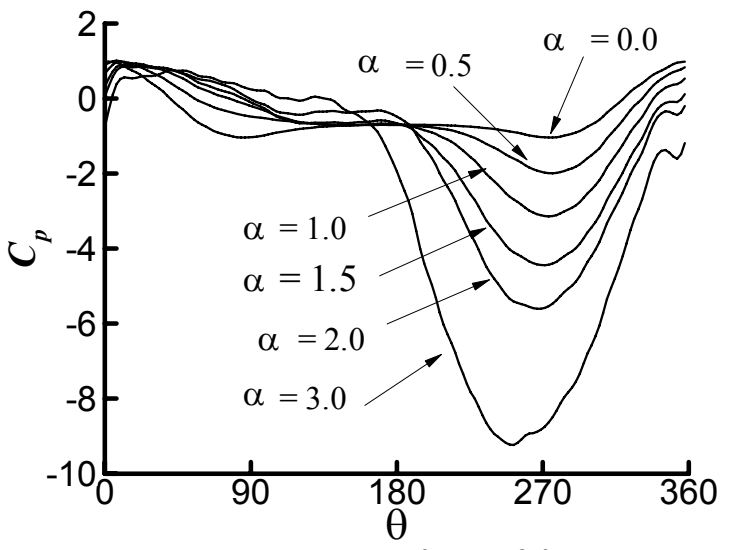

Figure 13. Pressure coefficient for $0 \leq \alpha \leq 3.0$ and $\mathbf{R e}=\mathbf{2 0 0}$

The behavior of the pressure coefficient is related to the vortex shedding process. In the points of high velocity and small local pressure (points $B$ and its symmetric), the pressure coefficient is minimum. The pressure coefficients for rotating cylinder are smaller than the value obtained for stationary cylinder. The pressure coefficient has the maximum value (equal to unit) at $\theta=0^{\circ}$, while for rotating cylinder this point oscillates. It was verified at the upper side of the cylinder $\left(\theta \approx 270^{\circ}\right)$ that the local pressure decreases significantly as the specific rotation increases. This behavior is due to the fact that the flow becomes asymmetric as a consequence of the rotation. In other words, the pressure on the decelerated side of the flow (lower side of the cylinder) decreases, while on the accelerated side it increases (in modulus). These behaviors were also observed by Kang et al. (1999) for Reynolds number equal to 60 , 100 and 160. It is worth mention that when compressible flows are taken into account, the pressure disturbances caused by the vortex formation propagate until $\theta=0^{\circ}$, which forces the pressure to oscillate at that point, even for a stationary cylinder.

\section{Lift and Drag Coefficients}

Figure 14 shows the mean values of the dynamic coefficients for different specific rotations. It can be verified that as the specific rotation increases, Fig. 14(a) shows that the mean values of lift coefficient increase, and Fig. 14(b) demonstrates that the drag coefficients decrease $(\alpha<2.0)$. On the other hand, for specific rotation greater than the critical value for each Reynolds number, the mean values of the drag coefficients increase. This effect is due to the capture of the fluid particles around the cylinder as illustrated in Fig. 15 that shows the streamlines around the cylinder for $R e=200$ and $\alpha=3.0$. According to Kang et al. (1999) this behavior differs significantly from those predicted using potential flow theory. For $\alpha=5.0$ it was obtained in the present work $C_{l}=18.4$. According to Mittal (2001), it can be expected higher values of $C_{l}$ in simulations at small Reynolds numbers and for twodimensional flows. The values obtained in the present work are lower than the value obtained using the potential flow theory $(2 \pi \alpha)$ and are greater than the proposed by Prandtl $(4 \pi)$. For the same Reynolds number and rotation ( $R e=200$ and $\alpha=5.0$ ), Mittal (2001) has found a mean lift coefficient equal to 27.5 . 

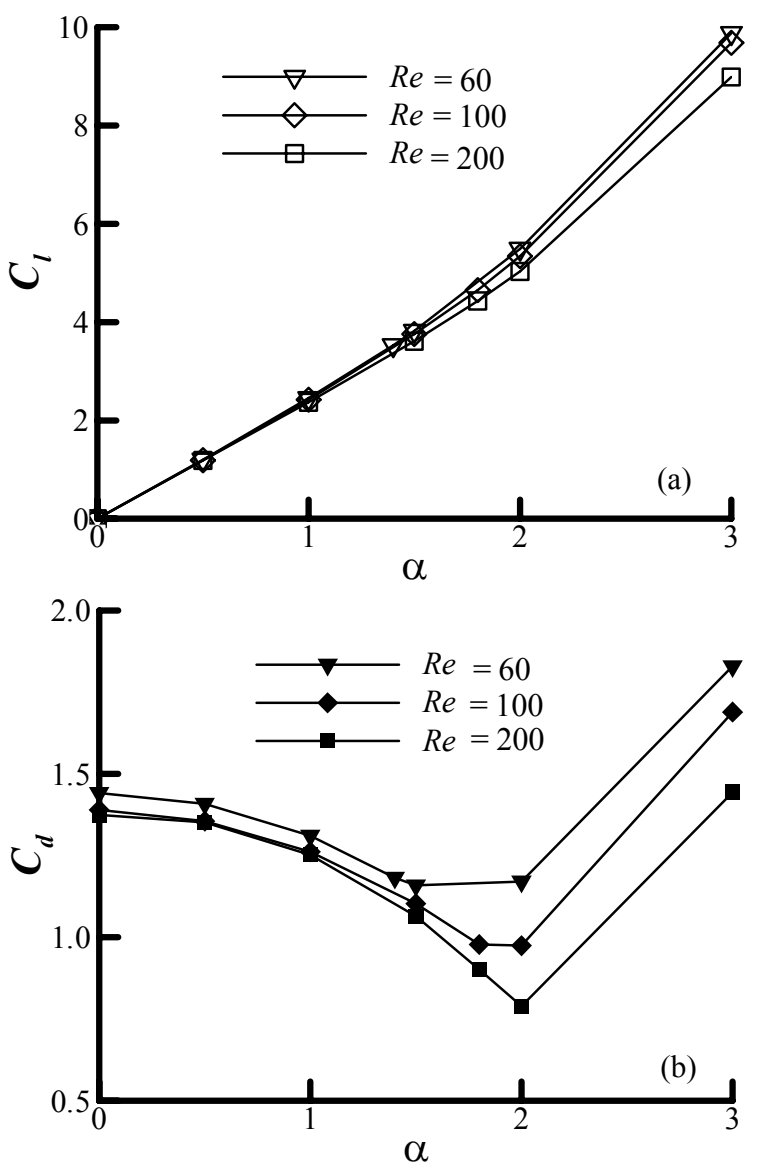

Figure 14. Mean values of the dynamics coefficients for $0 \leq \alpha \leq 3.0$ : (a) lift and (b) drag.

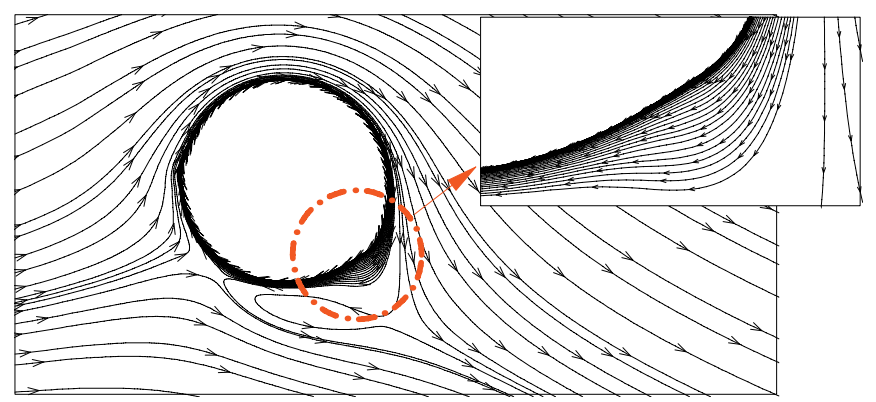

Figure 15. Illustration of the streamlines for $\operatorname{Re}=\mathbf{2 0 0}$ and $\alpha=\mathbf{3 . 0}$.

Figure 16 shows the root mean square of the drag and lift coefficients in comparison with the numerical results (Kang et al., 1999). It can be noted that the drag coefficient amplitude, Fig. 16(a), increases until a given value of $\alpha$ and, after, reduces, reaching an amplitude almost equal to zero. It is also observed that the amplitude increases as the Reynolds number and the rotation increases. On the other hand, the amplitude of the lift coefficient remains almost constant for $\alpha \leq 1.0$, for all Reynolds number, as shown in Fig. 16(b). For $\alpha>1.0$ the amplitude reduces and tends to be zero. From these figures, one can conclude that the influence of the rotation is more significant over the amplitude of the drag coefficient than the amplitude of the lift coefficient. Moreover, the present results are in agreement with the results presented by Kang et al. (1999).

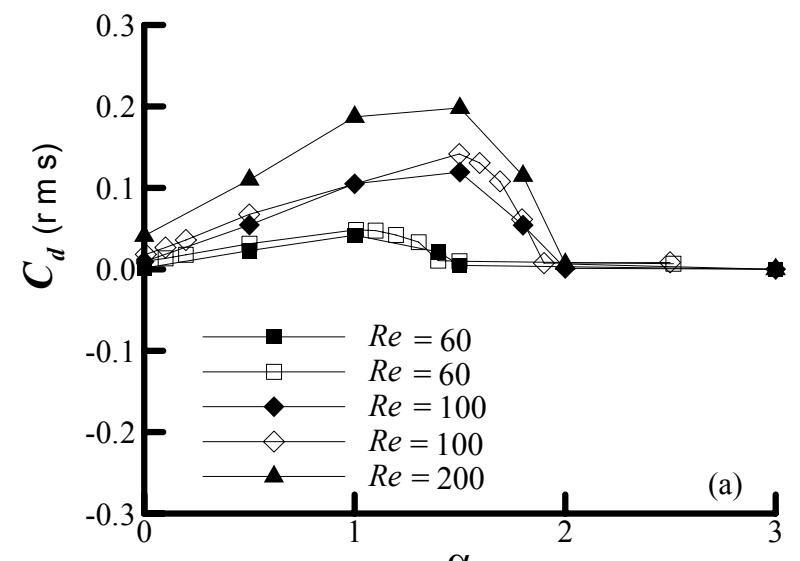

$\alpha$

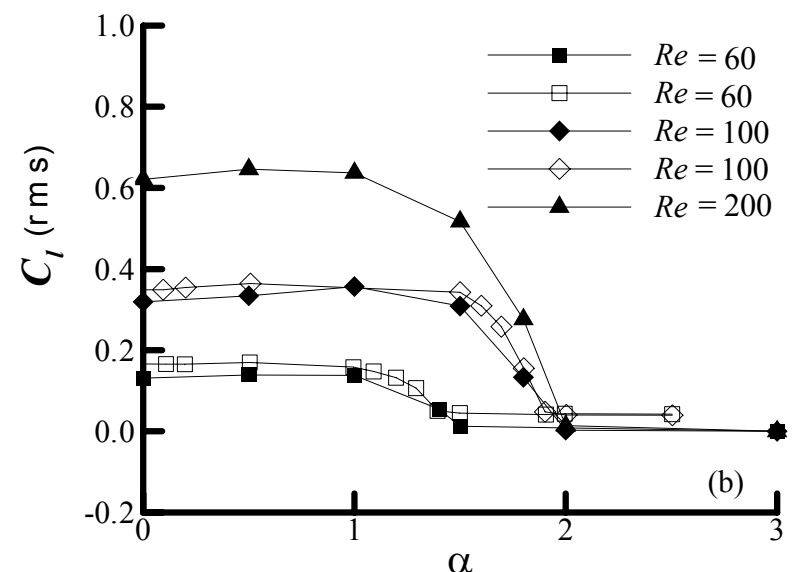

Figure 16. Root mean square of the dynamics coefficients for $0 \leq \alpha \leq 3.0$ : full symbols - present work; empty symbols - results by Kang et al. (1999).

\section{Vortex Shedding Frequency $(S t)$}

The values of Strouhal number obtained by Fast Fourier Transform (FFT) of the lift coefficient signal at Reynolds number equal to 60, 100 and 200 are shown in Fig. 17, and compared with the results by the literature. The time histories used for the analyses in the stationary case were greater than $200 \mathrm{~s}$, and for the rotation case, it was about $200 \mathrm{~s}$, in which the cycles of vortex shedding varied between 26 and 36 depending on the Reynolds number.

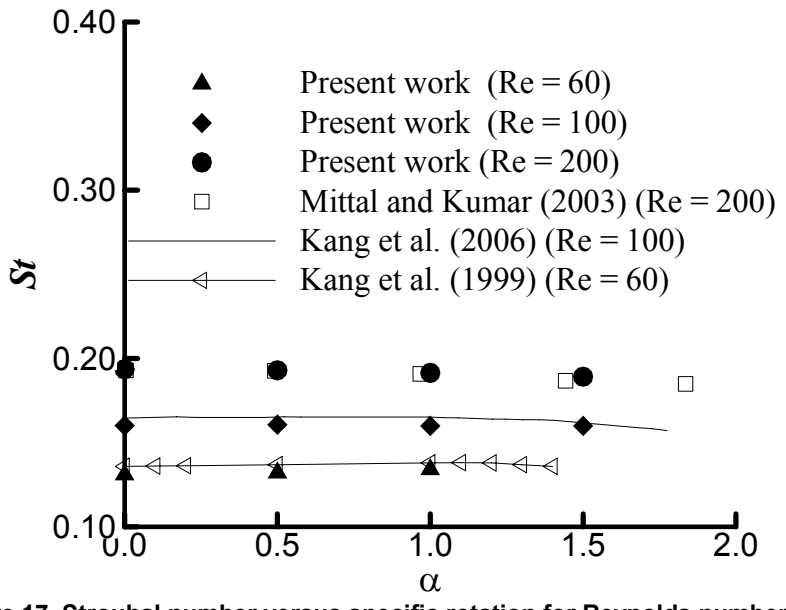

Figure 17. Strouhal number versus specific rotation for Reynolds numbers equal to 60,100 and 200 . 
It is important to note that for a small specific rotation ratio, the Strouhal number remains almost constant and it decreases as $\alpha$ approaches the critical value, and for $\alpha \geq \alpha_{c}$ it decreases abruptly to zero. Also, it can be seen that the Strouhal number is independent of the specific rotation and strongly dependent on the Reynolds number. As shown in Fig. 17, the values of Strouhal number increases with the Reynolds number.

\section{Concluding Remarks}

A numerical study of flows over a rotating circular cylinder has been performed using the Immersed Boundary Method (IBM) combined with the Virtual Physical Model (VPM). Flow fields, dynamic coefficients, pressure coefficients and Strouhal numbers, for a wide range of specific rotations, and for three values of Reynolds numbers, were presented. The main conclusions of the present work are: (i) the vortex shedding can be controlled by the rotation of the cylinder and it exists only for rotations smaller than the critical values, $\alpha_{c}$; (ii) the critical rotation is strongly dependent on the Reynolds number; (iii) the Strouhal number does not change significantly for small specific rotations, and reduces abruptly for $\alpha \geq \alpha_{c}$; (iv) the rotation of the cylinder is an efficient mechanism to reduce the drag force and to increase the lift force; (v) the $I B M$ combined with the $V P M$ can be easily employed for moving bodies.

Finally, the obtained results demonstrated the capability and the effectiveness of the numerical methodology used in this paper, and constitutes a promising tool to simulate more complex fluidstructure interaction problems. Also, one can take advantage of this work to better understanding the flow dynamic. In addition, it represents the continuity of a research project that is being developed at Federal University of Uberlândia.

\section{Acknowledgements}

The authors are deeply grateful to the following organizations:

- Minas Gerais State Agency FAPEMIG for the financial support to their research activities, especially through research project 509943/2010-1 and the pos-doctorate scholarship granted to A. R. da Silva.

- Brazilian Research Council - CNPq for the continued support to their research works.

- CAPES Foundation, from the Brazilian Ministry of Education.

\section{References}

Alam, Md.M. and Zhou, Y., 2007, "Flow Around Two Side-by-Side Closely Spaced Circular Cylinders", Journal of Fluids and Structures, Vol. 23, No. 5, pp. 799-805

Aldoss, T.K. and Abou-Arab, T.W., 1990, "Experimental Study of the Flow Around a Rotating Cylinder in Crossflow", Experimental Thermal and Fluid Science, Vol. 3, No. 3, pp. 316-322.

Badr, H.M., Dennis, S.C.R. and Young, P.J.S., 1989, "Steady and Unsteady Flow Past a Rotating Circular Cylinder at Low Reynolds Numbers", Computers and Fluids, Vol. 17, No. 4, pp. 579-609.
Chorin, A., 1968, "Numerical Solution of the Navier-Stokes Equations", Mathematics of Computation, Vol. 22, No. 104, pp. 745-762.

Coutanceau, M. and Menard, C., 1985, "Influence of Rotation on the Near-Wake Development Behind an Impulsively Started Circular Cylinder", Journal of Fluid Mechanics, Vol. 158, pp. 399-446.

da Silva, A.R., Silveira-Neto, A., Rade, D.A., Francis, R. and Santos, E.A., 2009, "Numerical Simulations of Flows over a Pair of Cylinders at Different Arrangements Using the Immersed Boundary Method", Computer Modeling in Engineering and Science, Vol. 50, No. 3, pp. 285-303.

Deng, J., Ren, A-L., Zou, J-F. and Shao, X-M., 2006, "Threedimensional Flow Around two Circular Cylinders in Tandem Arrangement", Fluid Dynamics Research, Vol. 38, No. 6, pp. 386-404.

Dol, S.S., Kopp, G.A. and Martinuzzi, R.J., 2007, “The Suppression of Periodic Vortex Shedding from a Rotating Circular Cylinder", Journal of Wind Engineering and Industrial Aerodynamics, Vol. 96, No. 6, pp. 1164-1184.

Juncu, G., 2007, "A Numerical Study of Momentum and Forced Convection Heat Transfer Around Two Tandem Circular Cylinders at Low Reynolds Numbers. Part I: Momentum Transfer", International Journal of Heat and Mass Transfer, Vol. 50, No. 19, pp. 3788-3798.

Kang, S., 2006, "Laminar Flow over a Steadily Rotating Circular Cylinder under the Influence of Uniform Shear", Physics of Fluid, Vol. 18, No. 4, pp. 47-106.

Kang, S., Choi, H. and Lee, S.C., 1999, "Laminar Flow past a Rotating Circular Cylinder", Physics of Fluid, Vol. 11, No. 11, pp. 3312-3320.

Lai, M-C. and Peskin, C.S., 2000, "An Immersed Boundary Method with Formal Second-Order Accuracy and Reduced Numerical Viscosity", Journal of Computational Physics, Vol. 160, No. 2, pp. 705-719.

Lima e Silva, A.L.F., da Silva, A.R. and Silveira-Neto, A., 2007, "Numerical Simulation of Two Dimensional Complex Flows Around Bluff Bodies using the Immersed Boundary Method", J. Braz. Soc. Mech. Sci. \& Eng., Vol. 29, No. 4, pp. 379-387.

Meitz, H. L and Fasel, H. F., 2000, "A compact-different scheme for the Navier-Stokes equations in vorticity-velocity formulation", Journal of Computational Physics, Vol. 157, pp 371-403.

Mittal, S., 2001, "Control of Flow past Bluff Bodies using Rotating Control Cylinders", Journal of Fluids and Structures, Vol. 15, No. 2, pp. 291-326.

Mittal, S. and Kumar, B., 2003, "Flow Past a Rotating Cylinder", Journal of Fluid Mech., Vol. 476, pp. 303-334.

Nair, M.T., Sengupta, T.K. and Chauhan, U.S., 1998, "Flow Past Rotating Cylinders at High Reynolds Numbers using Higher Order Upwind Scheme", Computers \& Fluids, Vol. 27, No. 1, pp. 47-70.

Peskin, C.S., 1977, "Numerical Analysis of Blood Flow in the Heart", Journal of Computational Physics, Vol. 25, No. 3, pp. 220-252.

Peskin, C.S. and McQueen, D.M., 1994, "A General Method for the Computer Simulation of Biological Systems Interacting with Fluids", Proceedings of the SEB Symphosium on Biological Fluid Dynamics, Leeds, England, July 5-8.

Ryan, K., Pregnalato, C.J., Thompson, M.C. and Hourigan, K., 2004, "Flow-Induced Vibrations of a Tethered Circular Cylinder", Journal of Fluids and Structures, Vol. 19, No. 8, pp. 1085-1102.

Schneider, G.E. and Zedan, M.A., 1981, “A Modified Strongly Implicit Procedure for the Numerical Solution of Field Problems", Numerical Heat Transfer, Vol. 4, No. 1, pp. 1-19.

Su, S-W., Lai, M-C. and Lin, C-A., 2006, "An Immersed Boundary Technique for Simulating complex flows with rigid boundary", Computers \& Fluids, Vol. 36, No. 2, pp. 313-324.

Sumner, D., Richards, M.D. and Akosile, O.O., 2007, "Strouhal Number Data for Two Staggered Circular Cylinders", J. Wind Eng. Ind. Aerodyn., Vol. 96, No. 6, pp. 859-871.

Williamson, C.H.K., 1997, "Advances in our Understanding of Vortex Dynamics in Bluff Body", Journal of Wind Engineering and Industrial Aerodynamics, Vols. 69-71, No. 49, pp. 3-32. 